\title{
Self-selection in a randomized trial of web-based primary and secondary prevention alcohol brief intervention
}

\author{
Nicolas Bertholet ${ }^{*}$, Joseph Studer ${ }^{1}$, John A Cunningham², Jean-Bernard Daeppen', Gerhard Gmel', \\ Bernard Burnand ${ }^{3}$
}

From International Network on Brief Interventions for Alcohol and Other Drugs (INEBRIA) Meeting 2013 Rome, Italy. 18-20 September 2013

How much a randomized controlled trial (RCT) sample is representative/differs from its declared source population is a challenging question, with major implications with regard to generalizibility of results. The question is crucial for freely available web-based interventions tested in RCTs.

We compared participants in a primary/secondary prevention web-based alcohol brief intervention RCT to its source population. There is a mandatory army recruitment process in Switzerland at age 19 for men. Between 8.2010 and 7.2011, 12,564 men attended two recruitment centers (source population) and were asked to answer a screening questionnaire on substance use; of the 11,819 (94\%) who completed it, 7,034 (56\%) agreed to participate in a cohort with regular assessments. In 2012, irrespective of their drinking, cohort participants were invited to the RCT; 1,549 agreed to participate. Using chi-square and t-test, we compared screening data of RCT participants to other members of the source population with respect to weekly alcohol use, maximum number of drinks/occasion, Alcohol Use Disorders Identification Test (AUDIT), prevalence of binge drinking ( $\geq 6$ drinks per occasion at least monthly), unhealthy alcohol use ( $>210 \mathrm{~g}$ of ethanol/week and/or $\geq 1$ binge episode/month), and abstinence.

RCT participants $(n=1,549)$ drank less than other members of the source population $(n=10,270)$ : they reported a mean (SD) of 6.2(8.1) vs 7.4(10.7) drinks/ week, $\mathrm{t}=5.2, \mathrm{p}<.0001 ; 9.7(7.7)$ vs 10.3(9.1) maximum number of drinks/occasion, $\mathrm{t}=2.6, \mathrm{p}=.009$; and 6.6(4.4) vs 7.2(4.9) AUDIT scores, $\mathrm{t}=4.7, \mathrm{p}<.0001$. The prevalence of binge drinking was lower among RCT participants $\left(36.7 \%\right.$ vs $\left.43.9 \%, \chi^{2}=28.8, \mathrm{p}<.0001\right)$, as was unhealthy alcohol use $\left(37.1 \%\right.$ vs $44.1 \%, \chi^{2}=27.5$, $\mathrm{p}<.0001)$ and abstinence $\left(7.8 \%\right.$ vs $\left.9.7 \%, \chi^{2}=5.8, \mathrm{p}=0.016\right)$. RCT participants differed from other members of the source population: they reported less drinking and lower levels of consequences or risk, but were also less likely to be abstainers, indicating that the self-selection applies to both ends of the drinking spectrum.

\section{Authors' details}

${ }^{1}$ Alcohol Treatment Center, Department of Community Medicine and Health, Lausanne University Hospital, Switzerland. ${ }^{2}$ Centre for Mental Health Research, Australian National University, Australia. ${ }^{3}$ Clinical Epidemiology Center, Department of Community Medicine and Health, Lausanne University Hospital, Switzerland.

Published: 4 September 2013

\section{doi:10.1186/1940-0640-8-S1-A10}

Cite this article as: Bertholet et al: Self-selection in a randomized trial of web-based primary and secondary prevention alcohol brief intervention. Addiction Science \& Clinical Practice 2013 8(Suppl 1):A10.

\footnotetext{
*Correspondence: Nicolas.Bertholet@chuv.ch

'Alcohol Treatment Center, Department of Community Medicine and Health, Lausanne University Hospital, Switzerland

Full list of author information is available at the end of the article
} 